\title{
Perivascular Space Semi-Automatic Segmentation (PVSSAS): A Tool for Segmenting, Viewing and Editing Perivascular Spaces
}

\author{
Authors: \\ Derek A Smith, BA ${ }^{\text {a,b,d }}$, (212) 824-8497, derekalexander.smith@mssm.edu \\ Gaurav Verma, PhD ${ }^{\text {a,b,d }}$, mahagaurav@gmail.com \\ Daniel Ranti, BS, ${ }^{\text {a,b,f }}$, (617) 645-8004, daniel.ranti@icahn.mssm.edu \\ Matthew Markowitz, PhD, ${ }^{\mathrm{g}}$, (212)413-3300, mmarkowitz@gradcenter.cuny.edu \\ Priti Balchandani, PhD ${ }^{a, b, c, d, e}$, (212) 241-0695, priti.balchandani@mssm.edu \\ Laurel Morris, PhD ${ }^{\text {a,b,d,f }}$, (212) 241-2774, laurel.morris@mssm.edu
}

Keywords: Perivascular Spaces, Semi-Automated, Frangi filter, Matlab, 7 T MRI, User-friendly

\#Pages: 8 \#Words: 1833 \#References: 30 \#Figs: 2 \#Tables: 0

Affiliation Addresses:

a Icahn School of Medicine at Mount Sinai, Hess CSM Building (1470 Madison Avenue, New York, NY, 10029)

${ }^{\mathrm{b}}$ BioMedical Engineering and Imaging Institute at Mount Sinai School of Medicine, Leon and Norma Hess

Center for Science and Medicine, 1470 Madison Avenue, New York, NY 10029

c Fishberg Department of Neuroscience at the Icahn School of Medicine at Mount Sinai, 1 Gustave L. Levy Place, New York, NY 10029

d Department of Radiology at the Icahn School of Medicine at Mount Sinai, 1 Gustave L. Levy Place, Box 1234, New York, NY 10029

${ }^{\mathrm{e}}$ Estelle and Daniel Maggin Department of Neurology at the Icahn School of Medicine at Mount Sinai, 1

Gustave L. Levy Place, Box 1137, New York, NY 10029

f Department of Psychiatry at the Icahn School of Medicine at Mount Sinai, 1 Gustave L. Levy Place, Box 1230, New York, NY 10029

g Advanced Science Research Center at The Graduate Center of the City University of New York, 85 St.

Nicholas Terrace, New York, NY 10031

Corresponding Author:

Derek A Smith, BA a,e,g, (212) 824-8497, derekalexander.smith@mssm.edu 
Summary:

Objective-- In this study, we validate and describe a user-friendly tool for PVS tracing that uses a Frangi-based detection algorithm; which will be made freely available to aid in future clinical and research applications. All PVS detected by the semi-automated method had a match with the manual dataset and 94\% of the manual PVS had a match within the semi-automated dataset.

Methods-We deployed a Frangi-based filter using a pre-existing Matlab toolbox. The PVSSAS tool pre-processes the images and is optimized for maximum effectiveness in this application. A user-friendly GUI was developed to aid the speed and ease in marking large numbers of PVS across the entire brain at once.

Results-- Using a tolerance of $0.7 \mathrm{~cm}, 83 \%$ of all PVSs detected by the semi-automated method had a match with the manual dataset and $94 \%$ of the manual PVS had a match within the semi-automated dataset. As shown in figure 3, there was generally excellent agreement between the manual and semi-automated markings in any given slice.

Significance-- The primary benefit of PVSSAS will be time saved marking PVS. Clinical MRI use is likely to become more widespread in the diagnosis, treatment, and study of MS and other degenerative neurological conditions in the coming years. Tools like the one presented here will be invaluable in ensuring that the tracing and quantitative analysis of these PVS does not act as a bottle neck to treatment and further research.

Keywords: Perivascular Spaces, Semi-Automated, Frangi filter, Matlab, 7 T MRI, User-friendly 


\section{Introduction}

MRI has become a valuable tool in the last several years to aid in the characterization and treatment of neuropsychiatric and neurological diseases, with the advent of ultra-high field imaging (7 T) being of particular note (Wisse et al. 2015; Sladky et al. 2013; Kerchner et al. 2010). 7 T MRI provides exceptionally detailed high resolution structural images, which has enabled in vivo study of the progression of neurodegenerative conditions (Liem et al. 2012; Tallantyre et al. 2010; Boutet et al. 2014). MRI has particularly become crucial in describing the presence and progression of perivascular spaces (PVS) (Patankar et al. 2005; Conforti et al. 2014; Groeschel et al. 2006; Kilsdonk et al. 2015; Wuerfel et al. 2008). PVS are small fluid-filled structures that appear on MRI scans in the vicinity of blood vessels. The presence and size of these PVS has been linked to gray and white matter pathology generally, and is of particular interest in a broad range of disorders like multiple sclerosis, cerebral small vessel disease, depression, and dementia (Rouhl et al. 2008; Favaretto et al. 2017; Zhu, Dufouil, et al. 2010; Zhu, Tzourio, et al. 2010; Heier et al. 1989; Patankar et al. 2007; Potter et al. 2015; Thomas et al. 2002). These PVS are believed to be strongly associated with the development and advancement of the disease and describing them in vivo is necessary to both clinical treatment of MS, and future research into potential treatments.

Manual detection and tracing of PVS is the current gold standard despite the fact that method is time consuming, prone to rater error/fatigue, and tends to underestimate the number of spaces present in the image. New methods using automated or user-assisted detection algorithms, particularly those using Frangi filters, have been explored and shown to be effective at reducing tracing time while improving accuracy and improving the number of PVS detected 
in both $3 \mathrm{~T}$ and $7 \mathrm{~T}$ environments (Dubost et al. 2018; Boespflug et al. 2018; Ballerini et al. 2018;

Park et al. 2016; Zhang et al. 2017; Zhang et al. 2016; Hou et al. 2017; Zong et al. 2016). In this study, we validate and describe a user-friendly tool for PVS tracing that uses a Frangi-based detection algorithm with added region growing features; which will be made freely available to aid in future clinical and research applications. $83 \%$ of PVS detected by the semi-automated method had a match with the manual dataset and $94 \%$ of the manual PVS had a match within the semi-automated dataset.

\section{Methods}

PVSSAS (Perivascular Space Semi-Automatic Segmentation) is a semi-automated tool for segmenting, viewing and editing PVS in the brain. ${ }^{1}$ PVSSAS runs on Matlab and is created using Matlabs inbuilt GUI editor, GUIDE. By default, PVS are segmented using a 2D frangi filter, but any custom segmentation can be used. Frangi filtering is a quick and powerful segmentation technique to segment vessel-shaped objects in 2 and 3 dimensions. Several papers have already applied frangi to 7T PVS segmentation in conjunction with other methods with fair success, although there is a lot of room for improvement (Ballerini et al. 2016; Zong et al. 2016; Hou et al. 2017).

An external frangi filter package made for Matlab is used (https://www.mathworks.com/matlabcentral/fileexchange/24409-hessian-based-frangivesselness-filter). Prior to application of the Frangi filter, the PVSSAS program pre-processes the T2-weighted structural images by applying a Gaussian burring function and normalizing the voxel intensity. PVSSAS also pre-processes the white matter mask by filling in small holes, under

\footnotetext{
${ }^{1}$ PVSSAS is available for download at: https://github.com/smithd37/pvssas
} 
200 voxels in size and performing a morphological erosion to remove potential false positives along the gray-white matter boundary. The Frangi filter is applied to the masked white matter region, using an adjustable setting sensitivity parameter (set to 0.5 standard deviations for the presented data). PVSs were also filtered by size to exclude candidates too small to be reliably identified or large but normal anatomical regions of CSF like ventricles. A range of 4 to 300 voxels was selected in consultation with radiologists and researchers on the T2-weighted imaging dataset.

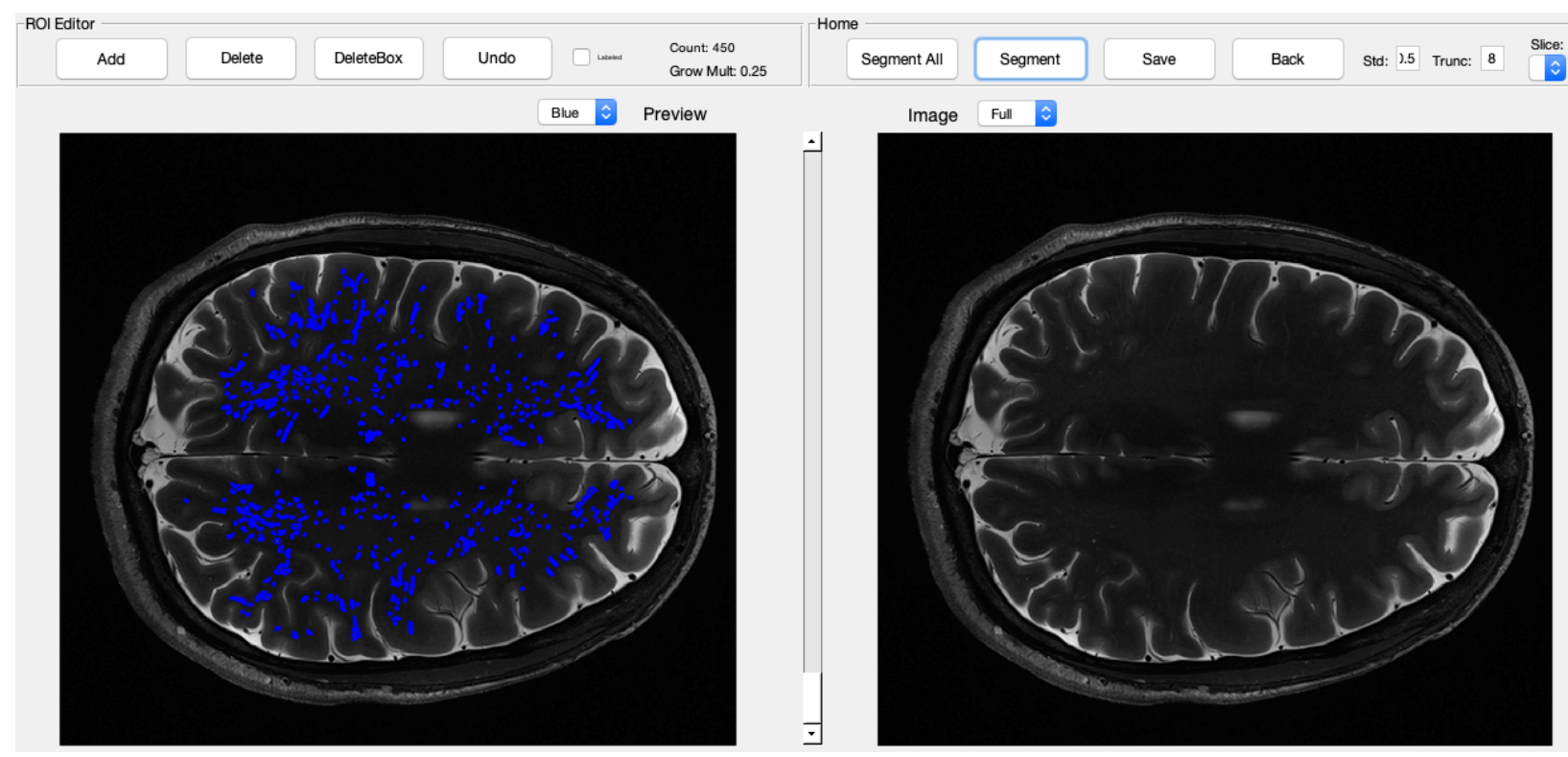

Figure 1. The primary interface for the PVSSAS tool. In the right view panel, the GUI displays raw images for the selected slice. On the toolbar, options are available for segmenting the whole brain, the selected slice, saving tracing masks, or for altering the parameters for the segmentation algorithm. In the left view panel, the completed segmentation can be viewed and edited - a trained reader can add or remove tracings.

Frangi parameters in pvssas_v1-4 were chosen among those that produced fair agreement with several manual markings, however it was noted that these marking under-estimated the number of PVS, and hence newer parameters may be needed. For pvssas_v5, parameters were 
changed slightly to resemble optimal parameters as determined by Ballerini, L. et al (Ballerini et al. 2018).

\section{$\underline{\text { Validation }}$}

PVSSAS was validated on a single multiple sclerosis patient, a male/female who was $n$ years old at the time of her scan. Our validation of PVSSAS was focused primarily on evaluating the relative speed, accuracy and sensitivity both manual and tool-assisted methods. In the manual method, markings were performed in Osirix by a trained MD with over 20 hours of experience. PVS were marked in the entire brain, which took approximately 6 hours to manually mark all 54 slices of the scan. ${ }^{2}$ Another rater was then trained to identify PVS on 7 T T2 TSE scans and also trained in use of the PVSSAS tool for 1-2 hours. The rater was told to focus on removing erroneous markings, not adding additional ones. It took approximately 30 minutes for the rater to completely mark the scan using PVSSAS. Separately, we assessed inter-rater reliability with two raters using PVSSAS, who focused on removing erroneous markings, not adding additional ones.

We focused our quantitative comparison of both methods on the sensitivity and specificity of each. This was done due to the fact that the manual tracings performed in Osirix marked each PVS with a cross-sectional point, rather than as volumes. Therefore, it was not possible to reliably compare other useful measures such as the relative length, average volume or total volumes of each. Sensitivity measures were determined by comparing the total number of PVS found using each method. Certain constraints were enforced: (1) manually traced PVS smaller than a single voxel were ignored, as these would be too small to be reliably detected by the

\footnotetext{
${ }^{2}$ Note: this includes certain areas that fall outside of the white-matter mask, and are therefore undetectable to the PVSSAS tool.
} 
PVSSAS; (2) our method was unable combine PVS that move between slices, so these spaces were counted as separate PVS in both the semi-automated and manually traced methods; (3) all PVS above voxels in size were ignored and this mostly affected semi-automated markings which were unlikely to be PVS; (4) all PVS that fell outside of the white matter mask were not used for comparison, as the semi-automated method is incapable of identifying them.

Specificity measures were determined by performing a slice-by-slice comparison between the semi-automated and manual tracings. For each slice the centroid (center voxel) of every tracing was determined using the RegionProps3 command in Matlab, and all centroids within $0.7 \mathrm{~cm}$ were considered successful overlap between the manual and semi-automated tracings. This number was determined based on an analysis of the number of PVS overlapping relative to the distance to the nearest match. Above $\sim 0.7 \mathrm{~mm}$, the distance to the closest match rapidly increases (fig. 2).
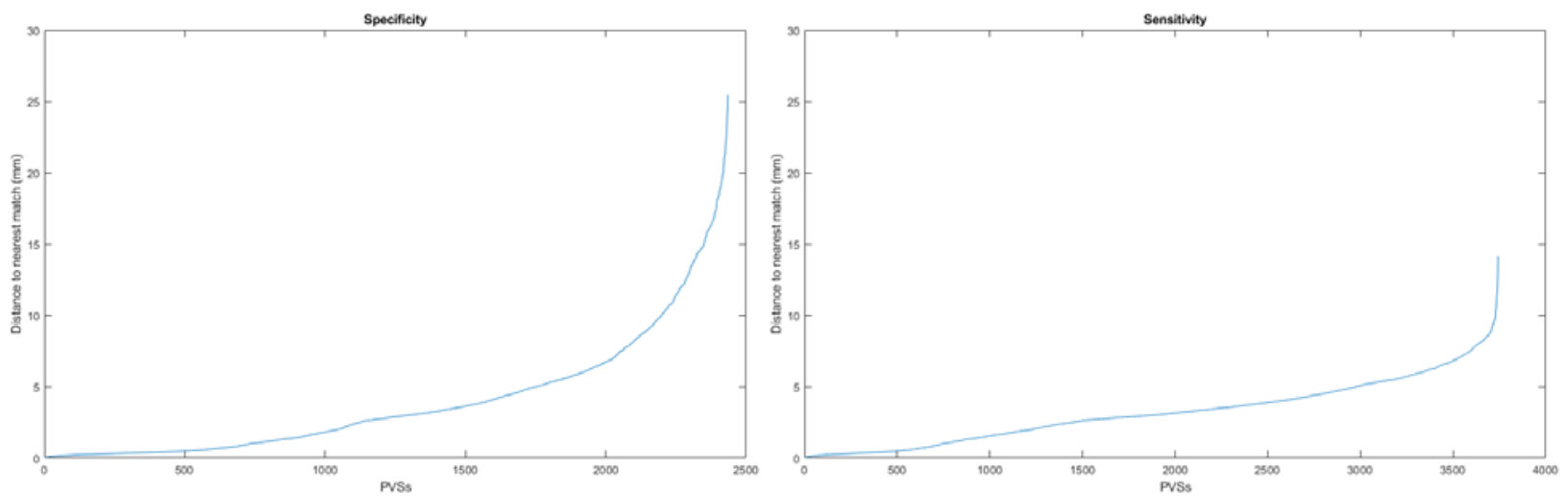

Figure 2. The Specificity (left) plot displays the relationship between the number of PVS found to be overlapping between the manual and semi-automated methods, relative to the distance to the nearest match. The Sensitivity (right) plot shows the number of PVS detected by the semiautomated method in relation to their nearest equivalent neighbors in the manual markings. 


\section{Results}

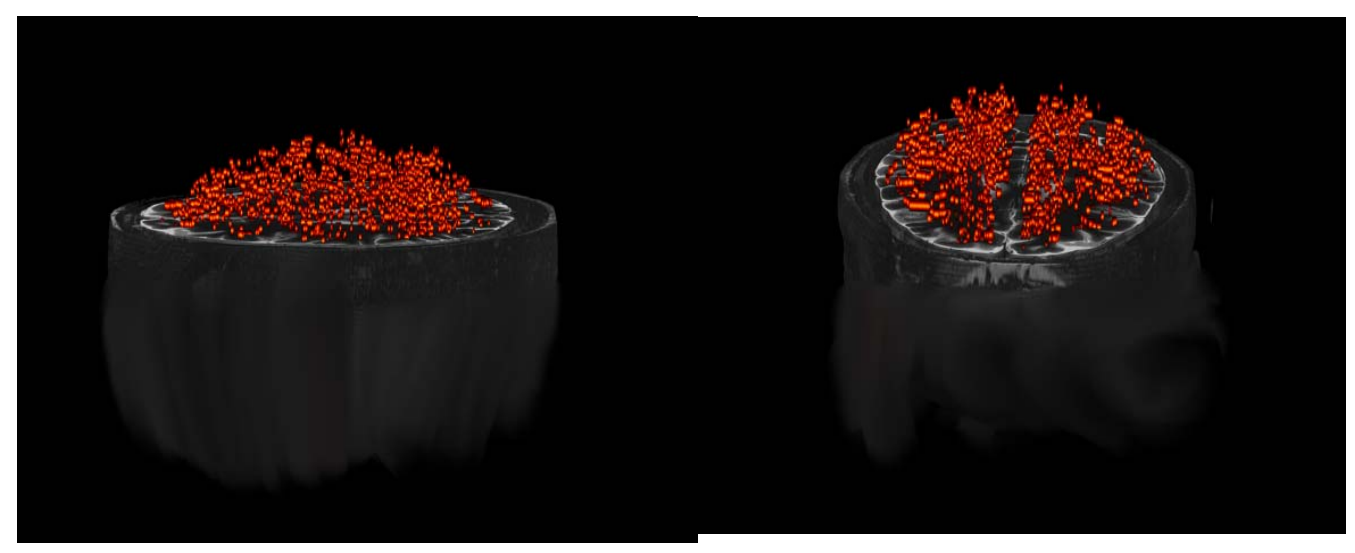

Figures 3\&4. Rendered 3-D view of total PVS found across all slices for one patient.

The semi-automated method identified a total of 2435 PVS. The manual reader identified 6162

PVS, but these included PVS outside the white matter mask. When applying the white matter

mask to the manual segmentation, the PVS count dropped from 6162 to 3743 . Using a

tolerance of $0.7 \mathrm{~cm}, 83 \%$ of all PVSs detected by the semi-automated method had a match with

the manual dataset and $94 \%$ of the manual PVS had a match within the semi-automated

dataset. As shown in figure 3, there was generally excellent agreement between the manual

and semi-automated markings in any given slice.

The inter-rater reliability between the two readers using the semi-automated method was as

follows: of the PVS identified by reader $1,97.8 \%$ of the PVS were in the identical location as

reader 2 , and $99.6 \%$ were within $0.8 \mathrm{~cm}$. Of the PVSs identified by reader $2,99.2 \%$ were in the same location as reader 1 , and $99.9 \%$ were within $0.8 \mathrm{~cm}$. Reader 1 identified 1680 PVSs, and reader 2 identified 1656 PVS. The Dice score between the two readers segmentations was

0.9914. 


\section{Discussion}

The primary benefit of PVSSAS will be time saved marking PVS. Using a tool such as PVSSAS, one rater with only a moderate amount of additional training to become familiarized with the UI can perform the same amount of total brain markings that currently requires multiple raters, at the combined total of 5-10+ man-hours per rater. Clinical MRI use is likely to become more widespread in the diagnosis, treatment, and study of MS and other degenerative neurological conditions in the coming years. Tools like the one presented here will be invaluable in ensuring that the tracing and quantitative analysis of these PVS does not act as a bottle neck to treatment and further research.

PVSSAS also provides valuable information that is currently not provided from other manual tracing programs, such as OsiriX. Typically, the process for marking an entire brain worth of PVS is very time consuming, and it is often unreasonable to expect raters to individually trace the entire volume of each PVS in an entire brain. However, the method used by PVSSAS automatically captures volume information for PVS, which could allow for more varied analysis of these spaces.

\section{Limitations}

In the current implementation of the PVSSAS there are certain restraints on both the sensitivity and specificity. The frangi filter used as the basis for PVSSAS is designed to look for objects at least 4 voxels in size. Similarly, PVSSAS has a maximum size of 100 voxels, although occasionally multiple traced objects in inadvertently overlap. Often this is easily spotted by raters, although it can affect the ability to reliably calculate the volume and centroid of certain PVS in a set of tracings. We recommend that users discount all tracings below 4 voxels and above 100 voxels 
bioRxiv preprint doi: https://doi.org/10.1101/2020.11.16.385336; this version posted November 17,2020 . The copyright holder for this preprint

(which was not certified by peer review) is the author/funder, who has granted bioRxiv a license to display the preprint in perpetuity. It is made available under aCC-BY-ND 4.0 International license.

as being erroneously marked, and focus on these areas as a good starting point for manually

marking the remainder of spaces. Even in spite of these limitation, the relatively high accuracy

and sensitivity of the tool means that it provides a reliable starting point for a rater to finish a

tracing much faster than a purely manual method.

Acknowledgements

Thank you to Ameen Al Qadi for his work in providing 3-D renderings for Figure's 3 and 4. 


\section{Bibliography}

Ballerini, L., Lovreglio, R., Hernández, M. del C.V., et al. 2016. Application of the ordered logit model to optimising frangi filter parameters for segmentation of perivascular spaces. Procedia Computer Science 90, pp. 61-67.

Ballerini, L., Lovreglio, R., Valdés Hernández, M.D.C., et al. 2018. Perivascular spaces segmentation in brain MRI using optimal 3D filtering. Scientific Reports 8(1), p. 2132. Boespflug, E.L., Schwartz, D.L., Lahna, D., et al. 2018. MR Imaging-based Multimodal Autoidentification of Perivascular Spaces (mMAPS): Automated Morphologic Segmentation of Enlarged Perivascular Spaces at Clinical Field Strength. Radiology 286(2), pp. 632-642.

Boutet, C., Chupin, M., Lehéricy, S., et al. 2014. Detection of volume loss in hippocampal layers in Alzheimer's disease using 7 T MRI: a feasibility study. Neurolmage. Clinical 5, pp. 341-348. Conforti, R., Cirillo, M., Saturnino, P.P., et al. 2014. Dilated Virchow-Robin spaces and multiple sclerosis: 3 T magnetic resonance study. La Radiologia Medica 119(6), pp. 408-414. Dubost, F., Adams, H., Bortsova, G., et al. 2018. 3D Regression Neural Network for the Quantification of Enlarged Perivascular Spaces in Brain MRI. arXiv.

Favaretto, A., Lazzarotto, A., Riccardi, A., et al. 2017. Enlarged Virchow Robin spaces associate with cognitive decline in multiple sclerosis. Plos One 12(10), p. e0185626.

Groeschel, S., Chong, W.K., Surtees, R. and Hanefeld, F. 2006. Virchow-Robin spaces on magnetic resonance images: normative data, their dilatation, and a review of the literature. Neuroradiology 48(10), pp. 745-754.

Heier, L.A., Bauer, C.J., Schwartz, L., Zimmerman, R.D., Morgello, S. and Deck, M.D. 1989. Large Virchow-Robin spaces: MR-clinical correlation. American Journal of Neuroradiology 10(5), pp. 929-936.

Hou, Y., Park, S.H., Wang, Q., et al. 2017. Enhancement of Perivascular Spaces in 7?T MR Image using Haar Transform of Non-local Cubes and Block-matching Filtering. Scientific Reports 7(1), p. 8569.

Kerchner, G.A., Hess, C.P., Hammond-Rosenbluth, K.E., et al. 2010. Hippocampal CA1 apical neuropil atrophy in mild Alzheimer disease visualized with 7-T MRI. Neurology 75(15), pp. 1381-1387.

Kilsdonk, I.D., Steenwijk, M.D., Pouwels, P.J.W., et al. 2015. Perivascular spaces in MS patients at 7 Tesla MRI: a marker of neurodegeneration? Multiple Sclerosis 21(2), pp. 155-162.

Liem, M.K., Lesnik Oberstein, S.A.J., Versluis, M.J., et al. 2012. 7 T MRI reveals diffuse iron deposition in putamen and caudate nucleus in CADASIL. Journal of Neurology, Neurosurgery, and Psychiatry 83(12), pp. 1180-1185.

Park, S.H., Zong, X., Gao, Y., Lin, W. and Shen, D. 2016. Segmentation of perivascular spaces in 7T MR image using auto-context model with orientation-normalized features. Neuroimage 134, pp. 223-235.

Patankar, T.F., Baldwin, R., Mitra, D., et al. 2007. Virchow-Robin space dilatation may predict resistance to antidepressant monotherapy in elderly patients with depression. Journal of Affective Disorders 97(1-3), pp. 265-270.

Patankar, T.F., Mitra, D., Varma, A., Snowden, J., Neary, D. and Jackson, A. 2005. Dilatation of the Virchow-Robin space is a sensitive indicator of cerebral microvascular disease: study in 
elderly patients with dementia. American Journal of Neuroradiology 26(6), pp. 1512-1520.

Potter, G.M., Doubal, F.N., Jackson, C.A., et al. 2015. Enlarged perivascular spaces and cerebral small vessel disease. International journal of stroke?: official journal of the International Stroke Society 10(3), pp. 376-381.

Rouhl, R.P.W., van Oostenbrugge, R.J., Knottnerus, I.L.H., Staals, J.E.A. and Lodder, J. 2008. Virchow-Robin spaces relate to cerebral small vessel disease severity. Journal of Neurology 255(5), pp. 692-696.

Sladky, R., Baldinger, P., Kranz, G.S., et al. 2013. High-resolution functional MRI of the human amygdala at 7 T. European Journal of Radiology 82(5), pp. 728-733.

Tallantyre, E.C., Morgan, P.S., Dixon, J.E., et al. 2010. 3 Tesla and 7 Tesla MRI of multiple sclerosis cortical lesions. Journal of Magnetic Resonance Imaging 32(4), pp. 971-977. Thomas, A.J., Perry, R., Barber, R., Kalaria, R.N. and O'Brien, J.T. 2002. Pathologies and pathological mechanisms for white matter hyperintensities in depression. Annals of the New York Academy of Sciences 977, pp. 333-339.

Wisse, L.E.M., Biessels, G.J., Stegenga, B.T., et al. 2015. Major depressive episodes over the course of 7 years and hippocampal subfield volumes at 7 tesla MRI: the PREDICT-MR study. Journal of Affective Disorders 175, pp. 1-7.

Wuerfel, J., Haertle, M., Waiczies, H., et al. 2008. Perivascular spaces--MRI marker of inflammatory activity in the brain? Brain: A Journal of Neurology 131(Pt 9), pp. 2332-2340. Zhang, J., Gao, Y., Park, S.H., Zong, X., Lin, W. and Shen, D. 2016. Segmentation of Perivascular Spaces Using Vascular Features and Structured Random Forest from 7T MR Image. Machine learning in medical imaging. MLMI (Workshop), author 10019, pp. 61-68.

Zhang, J., Gao, Y., Park, S.H., Zong, X., Lin, W. and Shen, D. 2017. Structured Learning for 3-D Perivascular Space Segmentation Using Vascular Features. IEEE Transactions on Bio-Medical Engineering 64(12), pp. 2803-2812.

Zhu, Y.-C., Dufouil, C., Soumaré, A., Mazoyer, B., Chabriat, H. and Tzourio, C. 2010. High degree of dilated Virchow-Robin spaces on MRI is associated with increased risk of dementia. Journal of Alzheimer's Disease 22(2), pp. 663-672.

Zhu, Y.-C., Tzourio, C., Soumaré, A., Mazoyer, B., Dufouil, C. and Chabriat, H. 2010. Severity of dilated Virchow-Robin spaces is associated with age, blood pressure, and MRI markers of small vessel disease: a population-based study. Stroke 41(11), pp. 2483-2490.

Zong, X., Park, S.H., Shen, D. and Lin, W. 2016. Visualization of perivascular spaces in the human brain at 7T: sequence optimization and morphology characterization. Neuroimage 125, pp. 895-902. 


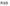
rape
telewer
now
wovias

te.

as $\mathrm{B}$ rave

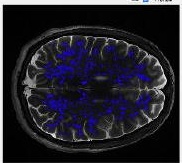

nos

int

tex

an thishers

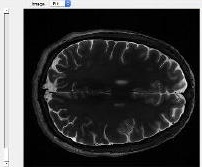

Ges. 


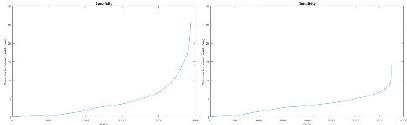




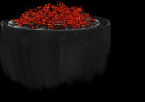




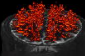

